\title{
흥 Tracking synapse-associated TCRs
}

To the editor:

We read with interest the article by Huppa et al. ${ }^{1}$ that shows prolonged and sustained interaction between T cells and antigenpresenting cells (APCs) is required for full T cell activation. As part of this study, the authors examined the spatial relationship between the T cell receptor (TCR)-CD3 complex and early signaling markers at the immunological synapse. TCR trafficking was monitored with immunofluorescence microscopic analysis of cyan fluorescent protein (CFP)-tagged $\mathrm{CD} 3 \zeta$ (CD3 $\zeta$-CFP), whereas TCR-derived signals (PI3K activity) were monitored by a yellow fluorescent protein (YFP)-tagged pleckstrin homology $(\mathrm{PH})$ domain of the serine-threonine kinase Akt (PH(AKT)-YFP). The authors showed rapid colocalization of $\mathrm{CD} 3 \zeta$-CFP and $\mathrm{PH}(\mathrm{AKT})$-YFP at the T cell-APC interface after B cell contact. Although PH(AKT)-YFP subsequently remained at the interface, $\mathrm{CD} 3 \zeta$-CFP was internalized into a central cluster of vesicles. In supplemental studies, the authors assessed TCR internalization by flow cytometric analyses of surface TCR $\beta$. These data showed rapid internalization of most TCRs after activation. The authors therefore concluded that removal of activated TCRs from the T cell-APC interface had no great effect on synapse-associated PI3K activity, but these data do not justify this conclusion.

The TCR is a multichain, heteromeric structure composed of a variable antigenbinding $(\alpha \beta)$ domain and noncovalently associated invariant signal-transducing complexes, the $\mathrm{CD} 3(\gamma, \delta$ and $\varepsilon)$ and $\zeta$ chains $^{2}$. Although loss of $\mathrm{CD} 3 \zeta$ chain from the $\mathrm{T}$ cell-APC interface is evident in the studies by Huppa et al., there are no data presented demonstrating a similar loss of TCRs from the immune synapse. This is important, given previous work showing surface $\mathrm{CD} 3 \zeta$ can partition to intracellular compartments independently of other TCR chains ${ }^{3-5}$. Furthermore, sustained exposure of $\mathrm{T}$ cells to antigen in vivo can induce the specific loss of $\mathrm{CD} 3 \zeta$ due to enhanced lysosomal degradation ${ }^{6}$. It is possible, therefore, that the dual-color, threedimensional video microscopy of sustained antigenic stimulation in the paper by Huppa and colleagues has in fact caught the $\mathrm{CD} 3 \zeta$ chain in the act of dissociating from the rest of the TCR.

Although we do not dispute the central conclusion of this paper, which documents very well the dynamics of synapse-associated CD3 $\zeta$-CFP after T cell-APC conjugate formation, the current data provide no insights into the dynamics of synapseassociated TCRs. This distinction, far from being trivial, will influence future analyses of molecular interactions among $\mathrm{T}$ cell and APC signaling proteins that partition to the immune synapse.

\section{Terri H Finkel}

Division of Rheumatology, The Children's Hospital of Philadelphia, University of Pennsylvania, Philadelphia, Pennsylvania 19104-4318, USA.

e-mail: finkelt@email.chop.edu

1. Huppa, J.B., Gleimer, M., Sumen, C. \& Davis, M.M. Nat. Immunol. 4, 749-755 (2003).

2. Ashwell, J.D. \& Klausner, R.D. Ann. Rev. Immunol. 8, 139-167 (1990).

3. Rozdzial, M.M., Malissen, B., Finkel, T.H. Immunity 3, 623-633 (1995).

4. Caplan, S., Zeliger, S., Wang, L. \& Baniyash, M. Proc. Natl. Acad. Sci. USA 92, 4768-4772 (1995).

5. Ono S., Ohno, H. \& Saito, T. Immunity 2, 639-44 (1995).

6. Bronstein-Sitton, N. et al. Nat. Immunol. 10 957-964 (2003).

\section{Davis replies:}

In our recent Nature Immunology paper $^{1}$ we suggested that after contact with peptide-major histocompatibility complex ligands presented by a B cell, "removal of activated TCRs from the contact interface had no appreciable effect on the amount of synapse-associated PI3K activity." Finkel disputes this conclusion because $\mathrm{CD} 3 \zeta$ can dissociate from the TCR in certain situations ${ }^{2}$.

Although $\mathrm{CD} 3 \zeta$ dissociation is a legitimate concern, it seems unlikely that this is an important factor in our study for two reasons. First, in an earlier paper we found that $\mathrm{CD} 3 \zeta$-GFP expression in a T cell line colocalized with TCR staining ${ }^{3}$ and thus it seems reasonable in most circumstances to use $\mathrm{CD} 3 \zeta$ and TCRs interchangeably. A second and stronger point is that in this manuscript, we showed by flow cytometry that approximately $70 \%$ of surface-localized TCR was internalized 30 minutes after initial B cell contact. This is in excellent agreement with the pool sizes of internalized and cell surface-localized $\mathrm{CD} 3 \zeta$-CFP that we quantified at this time point. Finkel notes that our flow cytometry results indicate a loss of surface TCR, but implies that this might not be due to internalization at the synapse. Only at the synapse, however, would TCRs contact their stimulatory ligands and be tagged for internalization and degradation ${ }^{4}$. Thus we have seen no evidence for any substantial separation of TCR from $\mathrm{CD} 3 \zeta$ in the T cells that we have studied.

\section{Johannes B Huppa \& Mark M Davis}

Department of Microbiology and Immunology and Howard Hughes Medical Institute, Stanford University School of Medicine, Stanford, California 94305, USA.

e-mail:mdavis@pmgm2.stanford.edu

1. Huppa, J.B., Gleimer, M., Sumen, C. \& Davis, M.M. Nat. Immunol. 4, 749-755 (2003).

2. Ono, S., Ohno, H. \& Saito, T. Immunity 2, 639-644 (1995).

3. Krummel, M.F., Sjaastad, M.D. Wulfing, C. \& Davis, M. M. Science 289, 1349-1352 (2000).

4. Lee, K.H. et al. Science 302,1218-1222 (2003). 